\title{
Passive ventricular constraint to improve left ventricular function and mechanics in an ovine model of heart failure secondary to acute myocardial infarction
}

\author{
James J. Pilla, PhD \\ Aaron S. Blom, BA \\ Daniel J. Brockman, BVSc \\ Victor A. Ferrari, MD \\ Qing Yuan, PhD \\ Michael A. Acker, MD
}

From the Departments of Surgery, Radiol-
ogy, and Medicine, University of Pennsyl-
vania Medical Center, Philadelphia, Pa.
Supported by a grant from Acorn Cardio-
vascular Inc, St Paul, Minn.
Received for publication June 6, 2002; re-
visions requested Aug 5, 2002; revisions
received Jan 7, 2003; accepted for publica-
tion Jan 22, 2003.
Address for reprints: Michael A. Acker,
MD, Associate Professor of Surgery, Hos-
pital of the University of Pennsylvania, 6th
Floor Silverstein Pavilion, Philadelphia, PA
19104.
J Thorac Cardiovasc Surg 2003;126:
1467-76
Copyright $\odot 2003$ by The American Asso-
ciation for Thoracic Surgery
0022-5223/2003 $\$ 30.00+0$
doi:10.1016/S0022-5223(03)00739-6

Objective: This study investigated the effects on global cardiac function and myocardial energetics of limiting progressive dilatation after infarction by means of a woven polyester jacket cardiac support device. We hypothesized that placement of the cardiac support device results in a decrease in myocardial wall stress and improvement in cardiac function and myocardial energetics.

Methods: To investigate the effect of passive constraint on left ventricular function and mechanics, a total of 10 sheep were studied with pressure-volume analysis and magnetic resonance imaging. A baseline study was followed by the creation of an anterior infarct. After 1 week, the animals underwent a second study. The cardiac support device was then placed over the epicardium in 5 sheep; the remaining animals served as controls. A terminal study was performed at 2 months after the infarct.

Results: The cardiac support device group at the terminal study exhibited a decrease in end-diastolic volume (control $110.3 \pm 19.8 \mathrm{~mL}$ vs cardiac support device $67.6 \pm$ $4.7 \mathrm{~mL}, P=.006$ ) and an improved ejection fraction (control $15.5 \% \pm 5.7 \%$ vs cardiac support device $29.46 \% \pm 4.42 \%, P=.008$ ) relative to the control group. Myocardial energetics were also enhanced in the cardiac support device group, as evidenced by the significant improvements in potential energy (control $2015 \pm 503$ $\mathrm{mL} \cdot \mathrm{mm} \mathrm{Hg} /$ beat vs cardiac support device $885 \pm 220 \mathrm{~mL} \cdot \mathrm{mm} \mathrm{Hg} /$ beat, $P=$ .006 ), efficiency (control $39.4 \% \pm 13.6 \%$ vs cardiac support device $59.8 \% \pm 8.5 \%$, $P=.044$ ), and oxygen consumption (control $0.072 \pm 0.013 \mathrm{~mL} \mathrm{O}_{2} /$ beat vs cardiac support device $0.052 \pm 0.007 \mathrm{~mL} \mathrm{O}_{2} /$ beat, $P=.034$ ).

Conclusion: Passive constraint with the cardiac support device after infarct prevents further remodeling and may stimulate reverse remodeling in heart failure secondary to acute myocardial infarction. These results suggest that in human beings placement of the cardiac support device after a large anterior myocardial infarction may be effective in halting the remodeling process that often leads to end-stage heart failure. If proved effective, placement of a cardiac support device after large heart attacks has the potential to decrease the incidence of heart failure that results after large myocardial infarctions.

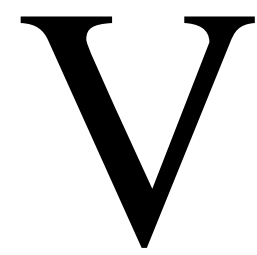

entricular remodeling after acute myocardial infarction (MI) is characterized by ventricular dilatation and functional impairment associated with an increased risk of heart failure. ${ }^{1-5}$ This process, characterized by significant architectural changes, such as loss of functional cardiac units, myocyte hypertrophy, and interstitial cellular fibrosis ${ }^{6}$ resulting from myocardial loss by infarction, remains incompletely understood. Recent studies suggest that left ventricular re- 
modeling is an important contributory event in the progression to end-stage congestive heart failure, ${ }^{7,8}$ and the degree of left ventricular enlargement has been identified as being associated with an unfavorable clinical outcome. ${ }^{9}$

The progressive deterioration in global cardiac function after MI is directly related to an increase in wall stress, which increases the workload of the left ventricle. Subsequently the heart enters into a positive feedback loop of progressive dysfunction that ultimately leads to end-stage heart failure. Pharmacologic therapy after MI, such as the use of $\beta$-blockers and angiotensin-converting-enzyme inhibitors, has been associated with significant improvement in cardiovascular mortality by curbing the deleterious effects of ventricular remodeling. ${ }^{10-12}$

In this study we investigated the effects on global cardiac function and myocardial energetics of mechanically limiting progressive myocardial dilatation after infarction by means of a bidirectional woven polyester jacket (Acorn Cardiac Support Device; Acorn Cardiovascular, Inc, St Paul, Minn). We hypothesized that placement of the cardiac support device (CSD) after infarction would result in a decrease in myocardial wall stress, with subsequent improvements in global cardiac function and myocardial energetics. Magnetic resonance imaging (MRI) and pressure-volume analysis (PVA) were used to investigate the effects of passive ventricular constraint in an ovine model of acute MI.

\section{Materials and Methods}

All animals used in this study received care in compliance with the Guide for Care and Use of Laboratory Animals prepared by the Institute of Laboratory Animal Resources, National Research Council, and published by the National Academy Press, revised 1996. The investigation was approved by the institutional animal care and use committee.

\section{Study Design}

To investigate the effect of limiting dilatation on myocardial function and energetics, a total of 10 Dorset sheep were studied with PVA and MRI. The experimental protocol for this study consisted of performing baseline PVA and MRI studies, followed by the creation of a transmural anterior wall MI. This was performed through a left thoracotomy by ligating the left anterior descending diagonal arteries. ${ }^{13}$ After a 1 -week recovery period, the animals underwent PVA and MRI studies again and were then randomly assigned to one of two groups, CSD or control. The custom polyester CSD was placed over the ventricular epicardium in 5 sheep through the same thoracotomy used for infarct creation. All animals received no further medical or surgical intervention for the duration of the study. Terminal PVA and MRI studies were performed at the 2-month postinfarct time point in both the control and CSD groups.

\section{Anesthesia}

One hour before surgery the animals were medicated with $1 \mathrm{mg} / \mathrm{kg}$ acepromazine and $0.001 \mathrm{mg} / \mathrm{kg}$ glycopyrrolate (INN glycopyrro- nium bromide). General anesthesia was induced with ketamine (10 $\mathrm{mg} / \mathrm{kg})$ and diazepam $(0.5 \mathrm{mg} / \mathrm{kg})$. Endotracheal intubation was performed, and the anesthesia was maintained with a mixture of $1 \%$ to $2 \%$ isoflurane in oxygen delivered by a time-cycled ventilator with a tidal volume of $20 \mathrm{~mL} / \mathrm{kg}$. Anesthesia was continuously adjusted and monitored to maintain a constant physiologic state in the animals. Postoperative analgesia consisted of flunixin meglumine $(2 \mathrm{mg} / \mathrm{kg})$ and buprenorphine $(2 \mu \mathrm{g} / \mathrm{kg})$ once immediately after extubation and every 4 hours for 48 hours. General anesthesia was used for all the surgical procedures and for the duration of the MRI studies.

\section{Animal Model}

The infarct model used in this study was originally characterized by Edmunds and colleagues ${ }^{13}$ at the University of Pennsylvania. The heart was exposed through a left thoracotomy through the fifth interspace with a partial fifth rib resection. On opening of the pericardium, the coronary anatomy was inspected to determine the vessels to be ligated. The criterion for ligation was any diagonal or obtuse margin vessel that supplied the anterior portion of the myocardium, excluding the left anterior descending coronary artery. Before ligation, the animals received a bolus of lidocaine as prophylaxis and were started on infusions of lidocaine and epinephrine. Systolic blood pressure was maintained at $80 \mathrm{~mm} \mathrm{Hg}$ throughout the procedure by means of successive bolus doses of phenylephrine. The vessels were then ligated with 3-0 Prolene (Ethicon, Inc, Somerville, NJ) suture. The chest remained open for an additional 45 minutes while the animal's vital signs and electrocardiogram were continuously monitored to ensure that the animal's condition was stable. After this period, in the 5 animals randomly assigned to the CSD group the pericardium was closed to minimize adhesions to the myocardium, whereas the pericardium in the remaining animals was left open. The incision was closed in layers, and a chest tube was placed to drain the chest and evacuate the pneumothorax (the chest tube was removed once the animal became ambulatory). The animal's vital signs and electrocardiogram were closely monitored for the initial 24-hour postoperative period before the animal was sent back to the colony.

\section{CSD Placement}

One week after infarction, the CSD was placed in 5 animals. This device is made of a custom-designed polyester weave that limits circumferential dilatation but allows longitudinal lengthening. With the animal under general anesthesia, the original thoracotomy was reopened and extended dorsally to allow resection of the remainder of the fifth rib. The pericardium was reopened to allow placement of the CSD, which was accomplished by sliding the device over the epicardium, up to the level of the atrioventricular junction. Prolene sutures (4-0) were placed along the base of heart starting on the posterior surface and working around anteriorly, with a total of between 8 and 10 sutures depending on the size of the heart. The excess material was gathered up along a line parallel with the long axis of the heart, excised, and resewn to ensure a snug final fit. The thoracotomy was closed as before, and a chest tube placed to evacuate the pneumothorax (and again removed when the animal became ambulatory). The animals received the same postoperative recovery measures as they did after the creation of the infarct. 


\section{PVA}

PVA was conducted with the animal under anesthesia as follows. A 7F multielectrode dual-field conductance catheter (Webster Labs, Baldwin Park, Calif) and a 5F micromanometer-tipped catheter (Millar Instruments, Inc, Houston, Tex) were placed under fluoroscopic guidance along the long axis of the left ventricular cavity through sterile cutdowns of the carotid and right femoral artery. Similarly, 20-mL occlusion catheters (Applied Vascular, Laguna Hills, Calif) were placed at the junction of the superior and inferior venae cavae with the right atrium through the right jugular and right femoral veins. A balloon-tipped pulmonary artery catheter was placed through the left jugular vein. Volume measurements were obtained with the conductance catheter technique. ${ }^{14-19}$ All hemodynamic signals and the electrocardiographic tracing were processed with an analog amplifier (Gould Electronics Inc, Eastlake, Ohio), digitized at $250 \mathrm{~Hz}$, and stored on computer disk for off-line analysis.

All data were collected with the ventilator held at end expiration. For determination of the end-systolic and end-diastolic pressure-volume relationships (ESPVR and EDPVR, respectively) ${ }^{20}$ and the preload-recruitable stroke work relationship (PRSW, the slope of stroke work vs end-diastolic volume [EDV]), ${ }^{21}$ the $20-\mathrm{mL}$ balloons in the venae cavae were gradually and temporarily inflated to alter preload as described previously elsewhere. ${ }^{19}$ All incisions were then closed primarily.

\section{MRI}

At baseline, 1 week, and 2 months after infarct, all animals underwent imaging in a 1.5-T whole-body high-speed clinical MRI system (GE Medical Systems, Milwaukee, Wis). Immediately after the PVA study, the animals were transported, still under general anesthesia, to the MRI unit. The left ventricular Millar catheter remained in place for the duration of the MRI study to allow MRI pressure-volume loops to be created by combining continuous left ventricular pressure data with MRI volume data. All images were cardiac and respiratory gated to ensure consistent spatial positioning of the heart during each acquisition. Tissue-tagged images in the short-axis plane were acquired for this study.

The imaging parameters were as follows: field of view $22 \mathrm{~cm}$, TR/TE 8.8/2.2 ms, slice thickness $6 \mathrm{~mm}$, interslice gap 0, tag spacing $5 \mathrm{~mm}$, 2 signal averages, and 6 to $8 \mathrm{k}$-space lines acquired per cardiac frame (depending on heart rate). Images were acquired in the short-axis plane with two $12.7-\mathrm{cm}$ surface coils placed on the left and right sides of the chest. The images were archived and stored for off-line analysis.

\section{Energetics}

Indices of myocardial energetics, ${ }^{22}$ including pressure-volume area (area bounded by the end-systolic elastance [Ees] and the pressure-volume loop), external work (area circumscribed by the pressure-volume loop), the potential energy (pressure-volume area minus external work), and myocardial oxygen consumption were obtained from combining the Ees obtained from the PVA with the volumes obtained by MRI. Myocardial mechanical efficiency was defined as the ratio of the external work to the total pressurevolume area.

\section{Statistics}

Statistical tests were performed with commercial computer software (SigmaStat; SPSS Inc, Chicago, Ill). All data are expressed as mean $\pm \mathrm{SD}$. Differences between time points within a group were assessed by 1-way analysis of variance with repeated measures followed by the multiple comparison method of Student-NewmanKeuls. Quantitative results between the two groups at the terminal time point were analyzed by $t$ test.

\section{Results}

Representative MRI images of a control sheep and a CSD sheep at the terminal time point are presented in Figure 1. These images were acquired at the midventricular level and at the end-diastole and end-systole points in the cardiac cycle. The images indicate that the CSD animal was less dilated and had a greater ejection fraction (EF) relative to the control sheep. In addition, the thinned area (as delineated by the arrows) appears to be less significant in the CSD animal.

Volume data obtained by MRI reveal that cardiac function deteriorated in the control group at the terminal time point. EDV in the control animals increased throughout the study, reaching significance $(P=.045)$ at the terminal time point relative to baseline (Figure 2). At the same time, EF decreased throughout the study, reaching significance at the terminal time point relative to both the baseline $(P=.01)$ and the 1 -week post infarct time points $(P=.01$; Figure 3$)$. All other volume parameters (stroke volume $P=.12$, cardiac output $P=.11$, and end-systolic volume [ESV]) degenerated at the terminal time point relative to 1 week after infarct, with only ESV achieving significance $(P=.005$; Table 1).

In the CSD group, EDV exhibited no significant change throughout the study, whereas EF decreased significantly from baseline to terminal but not from one-week to terminal. Stroke volume $(P=.23)$ and cardiac output $(P=.21)$ trended downward at the terminal time point, whereas ESV $(P=.67)$ remained virtually unchanged (Table 1$)$.

A comparison of the volume data at the terminal time point between the two groups showed that EDV and ESV were significantly reduced and EF was significantly improved in the CSD group. In addition, end-diastolic mass was significantly greater in the CSD group at 2 months after infarct (Table 1). This observed increase in mass is thought to be due to the CSD and adhesions, which cannot be differentiated from myocardium by MRI.

The load-independent indices Ees (Figure 4) and PRSW $(P=.17$; Table 1$)$ obtained by PVA trended downward in the control group, with Ees achieving significance $(P=$ .032). The remaining load-dependent indices $\mathrm{dP} / \mathrm{dt}_{\max }(P=$ $.051), \mathrm{dP} / \mathrm{dt}_{\min }(P=.051)$, and end-diastolic pressure $(P=$ .13) all trended toward decreased function but did not reach significance (Table 1).

In the CSD group the load-independent indices (Ees $P=$ .3 , PRSW $P=.2$ ) trended upward at the terminal point 

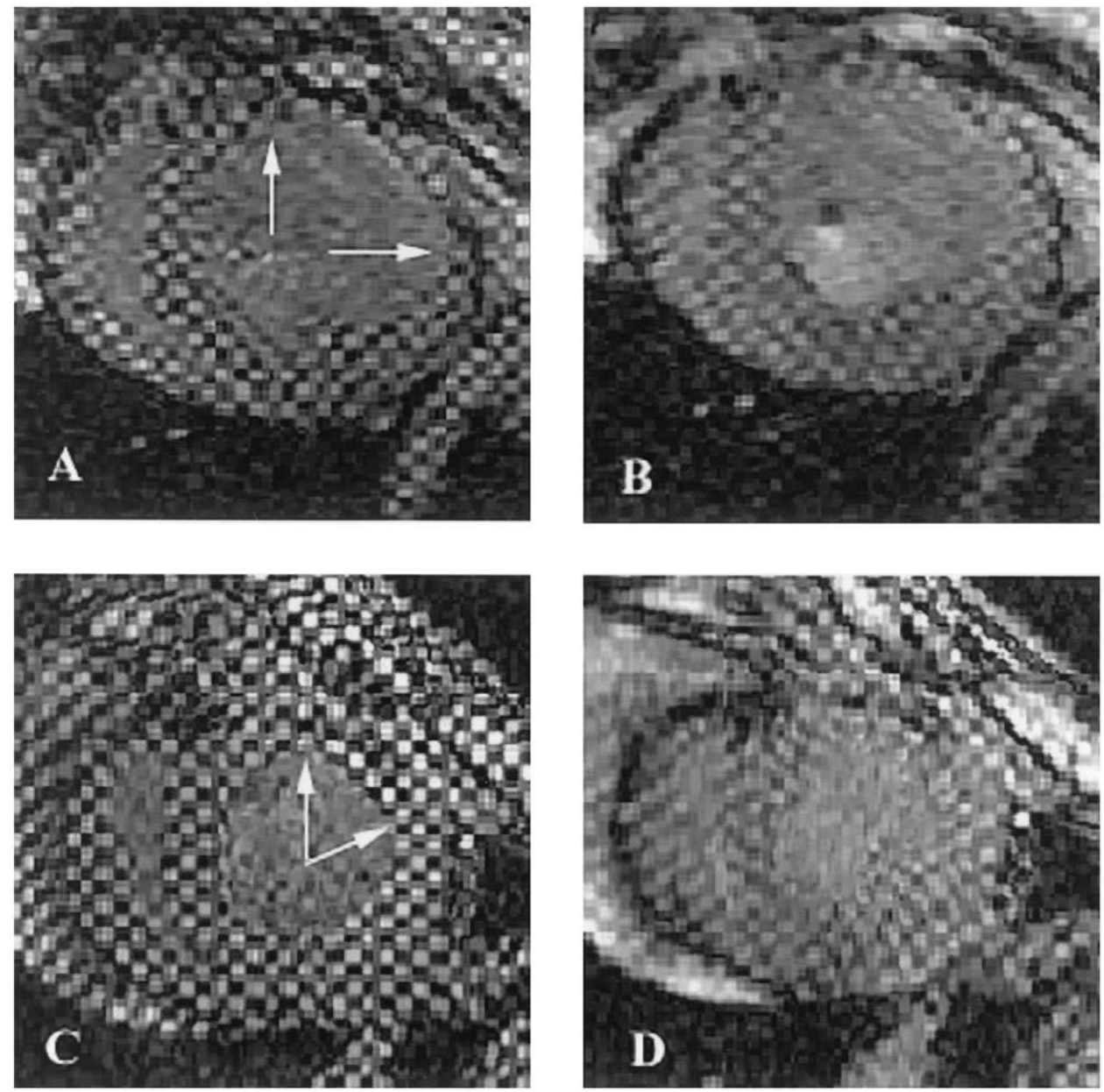

Figure 1. Representative short-axis MRI images of heart at level of midventricle. A, Control end-diastole. B, Control end-systole. C, CSD end-diastole. D, CSD end-systole. Control animals (A, B) have greater EDV and ESV, larger areas of thinned myocardium (arrows), and more abnormal geometry than CSD counterparts (C, D).

relative to the 1 -week point. In addition, $\mathrm{dP} / \mathrm{dt}_{\min }(P=.13)$ improved at the terminal time point but did not achieve significance. A comparison of the two groups at the terminal time point demonstrated that Ees was significantly $(P=$ .002) greater in the CSD group.

MRI-generated composite pressure-volume loops for the control and CSD groups are shown in Figure 5. The composite loops were generated by averaging the pressurevolume data at end-diastole, end-systole, end-isovolumic contraction, and end-isovolumic relaxation points for each animal in each group. In the control group there is a shift to the right from baseline to 1 week and from 1 week to terminal, indicating an increase in EDV and ESV. The CSD group exhibits the same rightward shift from baseline to 1 week, but from 1 week to terminal there is no shift in volume, indicting that the CSD has halted the dilatation.

The myocardial energetics data demonstrate that the potential energy $(P=.028)$ and efficiency $(P=.031)$ deteri- orated significantly relative to the baseline levels in the control group, whereas there were no significant changes in the CSD group (Table 1). Relative to the control group, at the terminal time point the CSD group had significantly lower potential energy $(P=.006)$, PVA $(P=.037)$, and oxygen consumption $(P=.034)$ and had an improved myocardial efficiency $(P=.044$; Figure 6$)$.

\section{Discussion}

Dynamic cardiomyoplasty can be considered the precursor of passive prosthetic ventricular constraint used as a therapy for end-stage heart failure. Though originally conceived as a means by which a patient's own skeletal muscle would contract around the heart to augment stroke volume, animal and clinical results suggested that the actual dynamic effect was inconsistent and limited, despite highly consistent clinical benefit. Studies in a canine model of chronic dilated cardiomyopathy by Patel and coworkers ${ }^{23}$ suggested that 


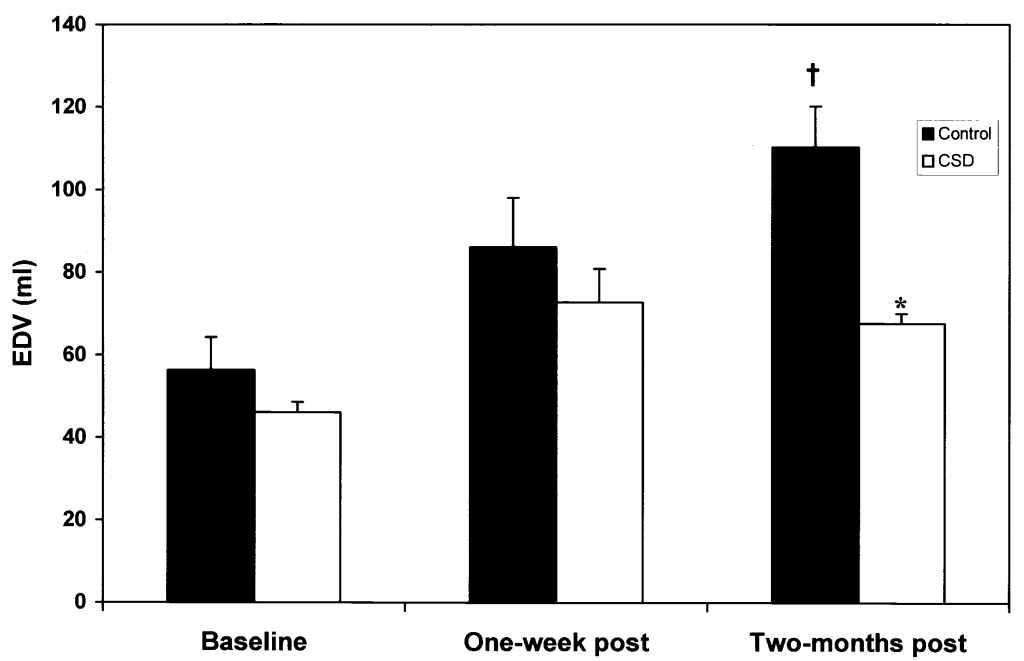

Figure 2. Graph depicting EDV changes. EDV was increased significantly in control group between baseline and 2-month time point. EDV was decreased significantly between the control and CSD groups at terminal time point. Dagger indicates $\boldsymbol{P}<.05$ between baseline and 2-month time points; asterisk indicates $\boldsymbol{P}<.05$ between control and CSD groups.

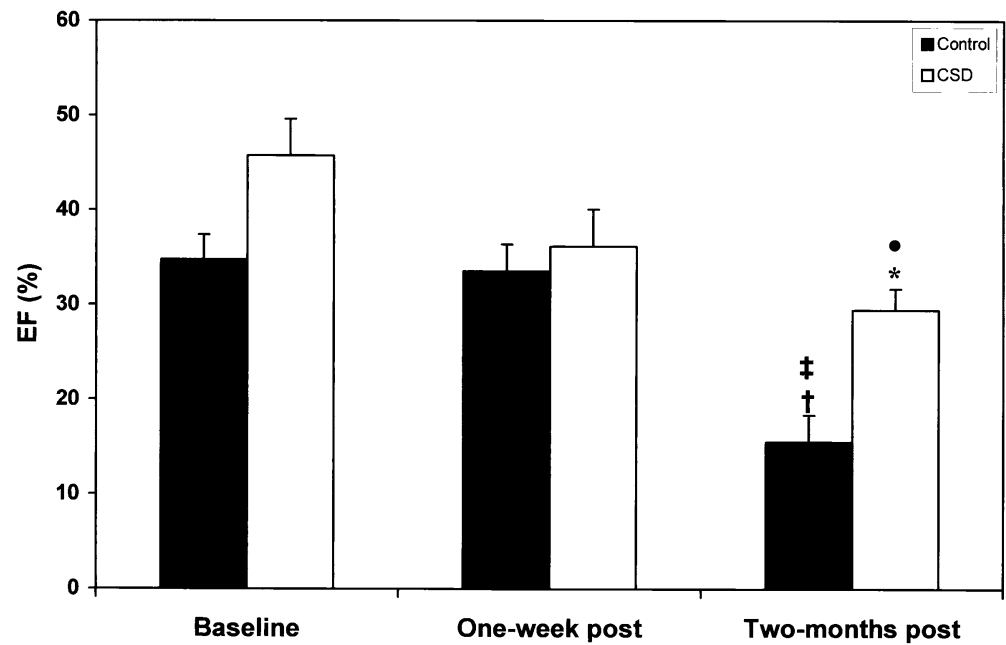

Figure 3. Graph depicting ejection fraction (EF) changes between control and CSD groups at baseline, 1 week after infarct, and 2 months after infarct. In control group there was significant decrease in ejection fraction between baseline and 2 months and 1 week and 2 months. In CSD group there was significant decrease in ejection fraction between baseline and 2-month time points. Finally, ejection fraction was increased significantly between control and CSD groups at terminal time point. Double dagger indicates $P<.05$ between 1-week and 2-month time points; dagger indicates $P<.05$ between baseline and 2-month time points; circle indicates $P<.05$ between baseline and 2-month time points; asterisk indicates $P<.05$ between control and CSD groups.

the chronic hemodynamic benefit after cardiomyoplasty was due to the passive effect of the skeletal muscle wrap around the heart alone, apart from any dynamic effect. This "girdling effect" of the conditioned muscle wrap was shown to stabilize the remodeling process of heart failure, arresting progressive deterioration of systolic and diastolic function. This observation prompted the development of a prosthetic jacket - the CSD - that would produce a girdling effect similar to that of the skeletal muscle wrap. Passive containment by an Acorn CSD was first studied by Chaudry and colleagues $^{24}$ in a well-established animal model of chronic dilated cardiomyopathy. They demonstrated that not only did hearts treated with the CSD not enlarge further, they actually decreased in volume and improved in function. In 


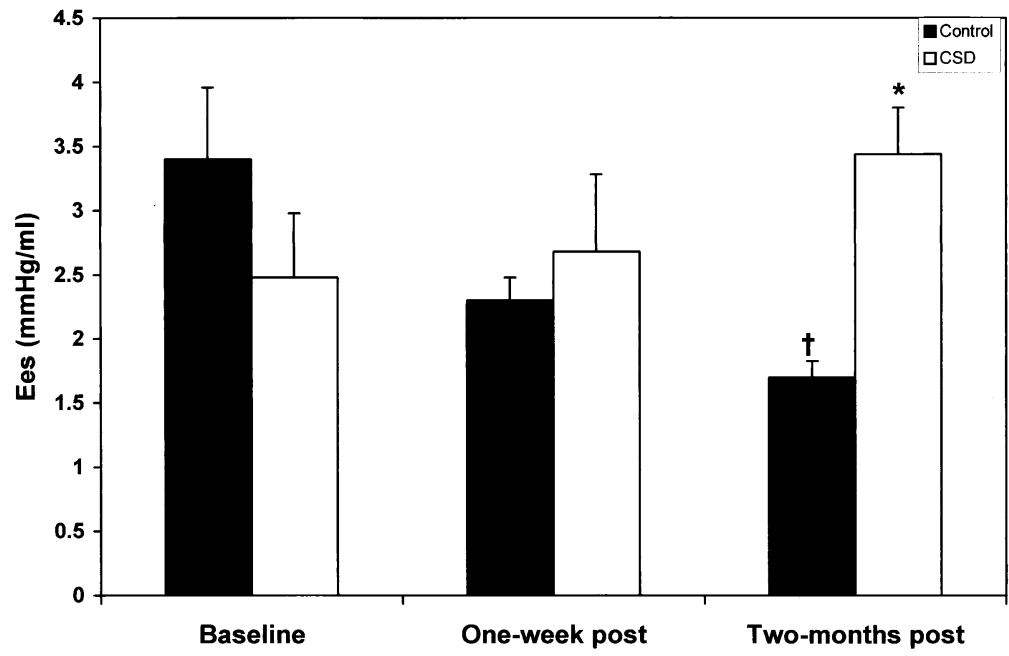

Figure 4. Graph depicting changes in end-systolic elastance (Ees). Elastance was decreased significantly in control group between baseline and 2 months. Elastance was increased significantly between control and CSD groups at 2-month time point. Dagger indicates $\boldsymbol{P}<.05$ between baseline and 2-month time points; asterisk indicates $P<.05$ between control and CSD groups.

TABLE 1. Load-dependent and load-independent PVA data, global function measures from MRI, and measures of myocardial energetics

\begin{tabular}{|c|c|c|c|c|c|c|}
\hline & \multicolumn{3}{|c|}{ Control } & \multicolumn{3}{|c|}{ CSD } \\
\hline & Baseline & 1 wk & Terminal & Baseline & 1 wk & Terminal \\
\hline \multicolumn{7}{|l|}{ PVA } \\
\hline Volume axis intercept (mL) & $-24.8 \pm 20.1$ & $11.2 \pm 12.0$ & $44.6 \pm 19.1^{*} \dagger$ & $-9.4 \pm 34.45$ & $3.0 \pm 22.6$ & $19.6 \pm 19.33$ \\
\hline ESPVR correlation & $0.991 \pm 0.005$ & $0.991 \pm .009$ & $0.990 \pm 0.008$ & $0.978 \pm 0.015$ & $0.988 \pm 0.009$ & $0.979 \pm 0.012$ \\
\hline PRSW (mm Hg) & $50.3 \pm 16.50$ & $45.11 \pm 15.78$ & $33.04 \pm 18.99$ & $55.57 \pm 13.00$ & $46.57 \pm 9.29$ & $60.8 \pm 22.39$ \\
\hline $\mathrm{dP} / \mathrm{dt}_{\max }(\mathrm{mm} \mathrm{Hg} / \mathrm{s})$ & $1492 \pm 235$ & $1457 \pm 434$ & $1069 \pm 211$ & $1407 \pm 404$ & $1232 \pm 461$ & $1538 \pm 355$ \\
\hline $\mathrm{dP} / \mathrm{dt}_{\min }(\mathrm{mm} \mathrm{Hg} / \mathrm{s})$ & $-1813 \pm 359$ & $-1306 \pm 255$ & $-1252 \pm 213$ & $-1509 \pm 368$ & $-1179 \pm 385$ & $-1517 \pm 251$ \\
\hline$\tau(\mathrm{ms})$ & $49.6 \pm 13.28$ & $74.13 \pm 21.93$ & $63.9 \pm 7.00$ & $57.9 \pm 16.25$ & $49.5 \pm 15.15$ & $54.8 \pm 12.62$ \\
\hline $\mathrm{EDP}(\mathrm{mm} \mathrm{Hg})$ & $5.25 \pm 1.86$ & $13.09 \pm 1.26$ & $10.54 \pm 2.24$ & $5.39 \pm 2.19$ & $8.92 \pm 1.3$ & $9.83 \pm 3.7$ \\
\hline Heart rate (beats/min) & $97.6 \pm 9.73$ & $104.8 \pm 10.98$ & $105.1 \pm 14.10$ & $94.6 \pm 13.01$ & $107.2 \pm 8.98$ & $111.2 \pm 9.86$ \\
\hline \multicolumn{7}{|l|}{ MRI } \\
\hline End-diastolic mass $(\mathrm{g})$ & $106.4 \pm 11.3$ & $112.6 \pm 25.23$ & $108.5 \pm 13.8$ & $112.2 \pm 14.2$ & $114.7 \pm 18.4$ & $144.07 \pm 4.9 \ddagger$ \\
\hline Stroke volume $(\mathrm{mL})$ & $20.2 \pm 8.76$ & $28.28 \pm 6.25$ & $17.72 \pm 9.97$ & $20.71 \pm 1.50$ & $27.15 \pm 11.46$ & $19.85 \pm 2.79$ \\
\hline Cardiac output (mL/min) & $2027 \pm 705$ & $2856 \pm 875$ & $1850 \pm 640$ & $1923 \pm 273$ & $2909 \pm 983$ & $2213 \pm 234$ \\
\hline End-systolic volume (mL) & $36.15 \pm 9.78$ & $57.5 \pm 18.48$ & $91.44 \pm 11.56^{*} \dagger$ & $30.81 \pm 11.70$ & $45.42 \pm 9.94$ & $47.77 \pm 5.63 \ddagger$ \\
\hline \multicolumn{7}{|l|}{ Energetics } \\
\hline $\begin{array}{l}\text { Potential energy (mL } \cdot \mathrm{mm} \\
\text { Hg/beat) }\end{array}$ & $1024 \pm 345$ & $1426 \pm 534$ & $2015 \pm 503 \dagger$ & $2149 \pm 1344$ & $1526 \pm 1116$ & $885 \pm 220 \ddagger$ \\
\hline Efficiency $(\%)$ & $60.8 \pm 12.0$ & $59.6 \pm 4.84$ & $39.4 \pm 13.6^{*} \dagger$ & $46.6 \pm 10.1$ & $60.1 \pm 10.1$ & $59.8 \pm 8.5 \ddagger$ \\
\hline
\end{tabular}

Volume axis intercept was increased significantly in the control group between baseline and terminal and between 1 week and terminal. End-diastolic mass was increased significantly between control and CSD groups at the 2-month time point. End-systolic volume was increased significantly between baseline and terminal and between 1 week and terminal in the control group but was decreased significantly between the control and CSD groups at terminal. Potential energy was increased significantly between baseline and terminal in the control group but was decreased significantly between control and CSD groups at terminal. Efficiency was decreased significantly between baseline and 1 week and between 1 week and terminal in the control group, whereas it was increased significantly between the control and CSD groups at terminal.

${ }^{*} P<.05$ between baseline and terminal.

$\dagger P<.05$ between 1 week and terminal.

$\ddagger P<.05$ between control and CSD groups.

addition to evidence of reverse remodeling on a ventricular level relative to control hearts, they further demonstrated that there was reversal of remodeling on a cellular level (single myocyte studies) and on a molecular level (a decrease in stretch proteins and improved calcium ion cycling). ${ }^{24}$ 
The first Acorn CSDs were placed in human beings in Germany in 1999. Since that time nearly 100 patients with dilated cardiomyopathies have had the CSD placed. So far it has been shown to be safe. In the few patients followed up for longer than 2 years, there have been no reports of constriction developing or of other major device-related complications. ${ }^{25}$ Adhesions after placement of a CSD around the heart have in a few cases necessitated transplantation, but in other cases adhesions have not been significant. In all cases transplantation was performed successfully. The presence of a CSD would make future surgical revascularization difficult. Although many patients have shown significant improvement, efficacy has yet to be demonstrated. There is an on-going prospective, randomized, multi-institutional study in both the United States and Europe to study the effectiveness of the CSD in patients with heart failure and dilated hearts.

This is the first study to indicate that passive ventricular constraint with the Acorn CSD prevents progressive LV dilatation after an acute MI. This is demonstrated by the fact that left ventricular pressure-volume loops generated for the control shifted rightward throughout the study, whereas after placement of the CSD the loops stabilized (Figure 6). This finding and other data presented in this study reveal that the CSD has halted the progressive remodeling seen after an acute MI. Further, the results also indicate that there may be some degree of reverse remodeling, as evidenced by an improvement in elastance and in the working conditions of the heart.

In this study we also examined the effects of CSD placement on myocardial energetics. At the terminal time point the CSD group had increased contractility (Figure 4), wasted less energy as heat, and consumed less oxygen. In contrast, the control group contracted less well while using more oxygen and wasting more energy as heat. Therefore for a given level of external work (similar to stroke work), the CSD group performed more efficiently and consumed less oxygen (Figure 6).

An improvement in function occurred in the absence of any evidence of restrictive or constrictive physiologic effect. This is supported by the diastolic function results (Table 1). End-diastolic pressure trended upward in both groups, and there was no significant difference between the groups at the terminal time point. The load-dependent measure of relaxation $\mathrm{dP} / \mathrm{dt}_{\text {min }}$ deteriorated in the control group throughout the course of the study, whereas in the CSD group this index improved at the terminal time point. The time constant of relaxation $(\tau)$ showed no significant difference between the two groups at the terminal time point.

The mechanism of action of the CSD is postulated to be that it limits myocardial wall stress by stabilizing the ventricular radius. The CSD may in fact not only limit but decrease wall stress by "off loading" a portion of the stress to the CSD
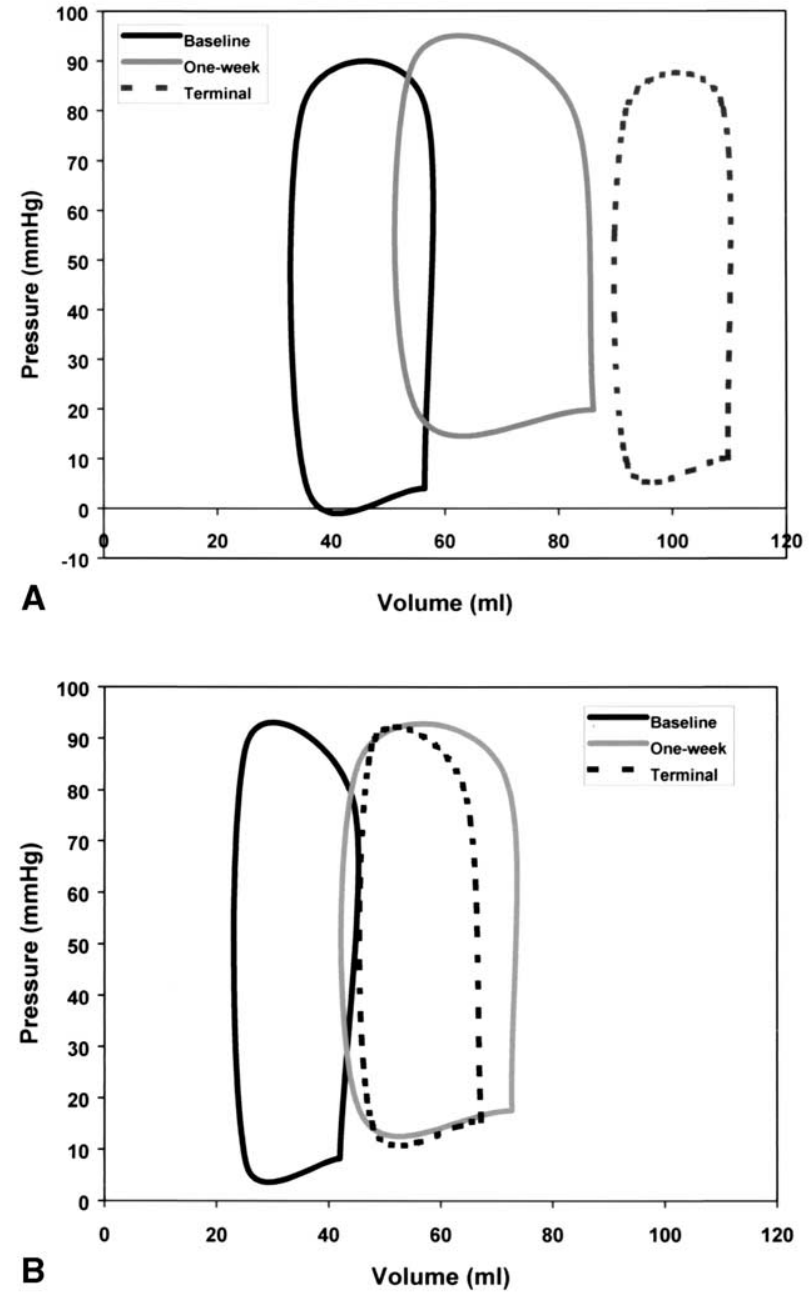

Figure 5. MRI-generated composite pressure-volume loops from control (A) and CSD (B) animals. Rightward shift of loops in control group demonstrates increase in EDV and ESV and deterioration in cardiac function between baseline and terminal time points. CSD loops show increase in volumes between baseline and 1 week but stabilization in function (as shown by leftward shift) at terminal time point.

(Figure 7). The sum of the forces without the CSD has the myocardium solely opposing the internal pressure. With the CSD in place, the same internal pressure is now opposed not only by the myocardium but also by the CSD, thus reducing the stress on the myocardium. This reduction in wall stress may stimulate the process of reverse remodeling. In both the chronic model of dilated cardiomyopathy and our model of remodeling after an acute MI, it is hypothesized that the CSD acts to decrease wall stress (global and regional). This acts as the stimulus to limit progressive remodeling and allows a degree of reverse remodeling.

The results of this study are consistent with a recent animal study that used a patch of Marlex (Phillips Petro- 
A. Stroke Work

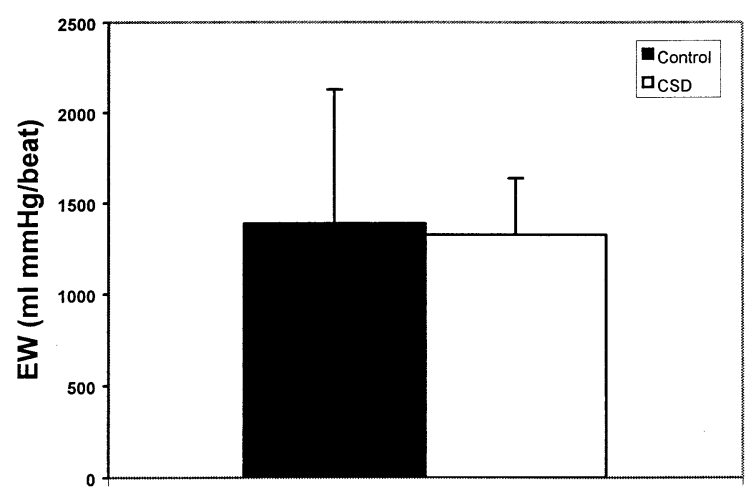

Two-months post

C. Efficiency

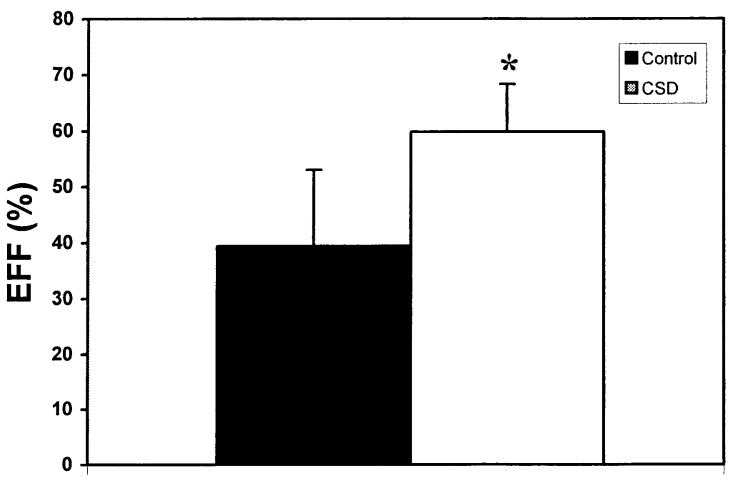

Two-months post
B. Potential Energy

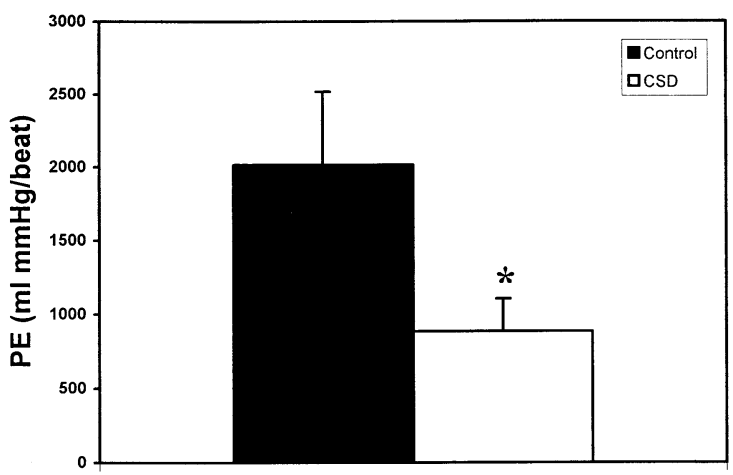

Two-months post

D. Oxygen Consumption

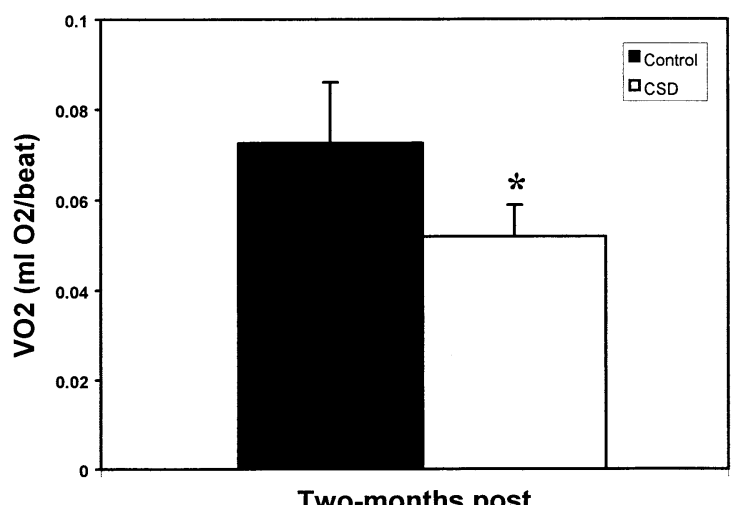

Figure 6. A, Stroke work (EW), at 2-month time point. B, Potential energy (PE, measure of heat loss from work) at 2-month time point. C, Efficiency (EFF) at 2-month time point. D, 0xygen consumption (VO2) at 2-month time point. Potential energy and oxygen consumption were significantly decreased between control and CSD groups at 2-month time-point. Efficiency was significantly increased between control and CSD groups at 2-month time-point. Asterisk indicates $P<.05$ between control and CSD groups.
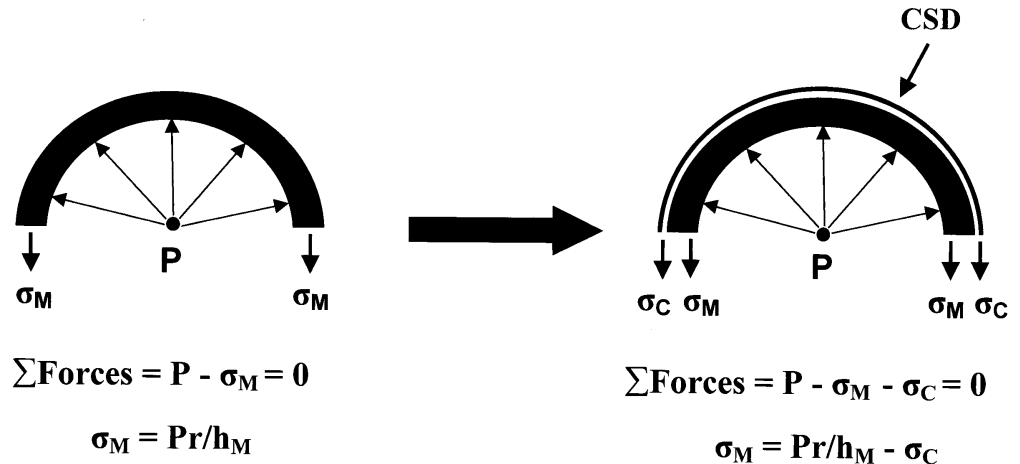

$$
\begin{gathered}
\sum \text { Forces }=P-\sigma_{M}-\sigma_{C}=0 \\
\sigma_{M}=\operatorname{Pr} / h_{M}-\sigma_{C}
\end{gathered}
$$

Figure 7. Forces on cross-section of myocardium depicted without CSD (A) and with CSD (B). Sum of forces without CSD results in internal pressure being opposed solely by stress in myocardium. With CSD placement internal pressure is opposed by stress in myocardium and stress on CSD, thus reducing myocardial wall stress. Assumes an isotropic homogeneous sphere at static equilibrium. 
leum Company, Bartlesville, Okla) mesh applied before the creation of an infarct. ${ }^{26}$ Their findings were also attributed to the limiting of myocardial wall stress, which they postulated preserved the border zone to result in a stable, nonexpanding infarct.

There are some limitations to this study that merit consideration. The pericardium on the CSD sheep was closed immediately after infarct to facilitate CSD placement the next week, whereas the pericardium was left opened in the control group. This does not correlate with the clinical situation and theoretically may result in increased remodeling in the control animals because pericardial restraint has been removed. However, at 1 week after infarct there was no statistical difference between the two groups, indicating that closing the pericardium had no observable effect on function and that both groups had remodeling to an equal degree. Another limitation is the lack of a sham operation at 1 week on the control animals. The effect of a sham operation, if anything, however, would be a negative one on the control group. Additional studies are also need to assess the efficacy of the CSD for longer periods in animals with advanced heart failure secondary to acute MI and to more clearly elucidate the cellular and molecular mechanism behind passive constraint and its benefit in heart failure.

In conclusion, passive constraint with the CSD after infarct prevents further remodeling and may stimulate reverse remodeling in sheep with heart failure secondary to acute MI. These results suggest that in human beings placement of the CSD after a large anterior MI may be effective in halting the remodeling process that often leads to endstage heart failure. If proved effective, placement of a CSD has the potential to decrease the incidence of heart failure that results after large MIs, but the final determination of whether these results can be extrapolated to human beings must wait for a randomized, controlled trail.

\section{References}

1. Eaton LW, Weiss JL, Bulkley BH, Garrison JB, Weisfeldt ML. Regional cardiac dilatation after acute myocardial infarction: recognition by two-dimensional echocardiography. $N$ Engl J Med. 1979;300:5762.

2. Pfeffer MA, Braunwald E. Ventricular remodeling after myocardial infarction: experimental observations and clinical implications. Circulation. 1990;81:1161-72.

3. McKay RG, Pfeffer MA, Pasternak RC, Markis JE, Come PC, Nakao $\mathrm{S}$, Alderman JD, et al. Left ventricular remodeling after myocardial infarction: a corollary to infarct expansion. Circulation. 1986;74:693702 .

4. Meizlisch JL, Berger HJ, Plankey M, Errico D, Levy W, Zaret BL. Functional left ventricular aneurysm formation after acute anterior transmural myocardial infarction: incidence, natural history and prognostic implications. N Engl J Med. 1984;311:1001-6.

5. Kim C, Braunwald E. Potential benefits of late reperfusion of infarcted myocardium: the open artery hypothesis. Circulation. 1993;88:242636.

6. Sabbah HN, Goldstein S. Ventricular remodeling: consequences and therapy. Eur Heart J. 1993;14:24-9.

7. Spinale FG, Zellner JL, Tomita M, Crawford FA, Zile MR. Relation- ship between ventricular and myocyte remodeling with the development and regression of supraventricular tachycardia induced cardiomyopathy. Circ Res. 1991;69:1058-67.

8. Tomita M, Spinale FG, Crawford FA, Zile MR. Changes in left ventricular volume, mass and function during development and regression of supraventricular tachycardia induced cardiomyopathy, disparity between recovery of systolic vs diastolic function. Circulation. 1991;83:635-44.

9. McKee PA, Castelli WP, McNamara PM, Kannal WB. The natural history of congestive heart failure: the Framington study. $N$ Engl J Med. 1971;385:1441-6.

10. Sabbah HN, Shimoyama H, Kono T, Gupta RC, Sharov VG, Scicli G, et al. Effects of long-term monotherapy with enalapril, metoprolol, and digoxin on the progression of left ventricular dysfunction and dilatation in dogs with reduced ejection fraction. Circulation. 1994;84: 2852-9.

11. Pfeffer MA, Launas GA, Vaughan DE, Parisi AF, Braunwald E. Effect of captopril on progressive ventricular dilatation after anterior myocardial infarction. N Engl J Med. 1988;319:80-6.

12. Waagstein F, Caidahl $\mathrm{K}$, Wallentin I, Bergh $\mathrm{CH}$, Hjalmarson A. Long-term $\beta$-blockade in dilated cardiomyopathy. Effects of shortand long-term metoprolol treatment followed by withdrawal and readministration of metoprolol. Circulation. 1989;80:551-63.

13. Gorman JH, Gorman RC, Plappert T, Jackson BM, Hiramatsu Y, St John-Sutton MG, et al. Infarct size and location determine development of mitral regurgitation in the sheep model. $J$ Thorac Cardiovasc Surg. 1998;115:615-22.

14. Baan J, Jong TT, Kerkhof PL, Moene RJ, van Dijk AD, van der Velde ET, et al. Continuous stroke volume and cardiac output from intraventricular dimensions obtained with the impedance catheter. Cardiovasc Res. 1981;15:328-34.

15. Baan J, van der Velde ET, de Bruin HG, Smeek GJ, Koops J, van Dijk $\mathrm{AD}$, et al. Continuous measurement of the left ventricular volume in animals and humans by the conductance catheter. Circulation. 1984; 70:812-23.

16. Steendjik P, van der Velde ET, Baan J. Left ventricular stroke volume by single and dual field excitation of conductance catheter in dogs. Am J Physiol.. 1993;264(6 Pt 2):H2198-207.

17. Kass DA. Clinical evaluation of left heart function by conductance catheter technique. Eur Heart J. 1992;13(Suppl E):57-64.

18. Burkhoff D. The conductance method of left ventricular volume estimation-methodologic limitations put into perspective. Circulation. 1990;81:703-6.

19. Kass DA, Yamazaki T, Burkhoff D, Maughan WL, Sagawa K. Determination of left ventricular end-systolic pressure-volume relationships by the conductance (volume) catheter technique. Circulation. 1986; 73:586-95.

20. Sagawa K, Maughan L, Suga H, Sunagawa K. Cardiac contraction and the pressure-volume relationship. London: Oxford University Press; 1988.

21. Glower DD, Spratt JA, Snow ND, Kabas JS, Davis JW, Olsen CO, et al. Linearity of the Frank-Starling relationship in the intact heart: the concept of preload recruitable stroke work. Circulation. 1985;71:9941009.

22. Suga H, Hayashi T, Shirahata M, Suehiro S, Hisano. Regression of cardiac oxygen consumption on ventricular pressure-volume area in dog. Am J Physiol.. 1981;240:H320-5.

23. Patel HJ, Polidori DJ, Pilla JJ, Plappert T, Kass D, St John Sutton M, et al. Stabilization of chronic remodeling by asynchronous cardiomyoplasty in dilated cardiomyopathy: effects of a conditioned muscle wrap. Circulation. 1997;96:3665-71

24. Chaudhry PA, Mishima T, Sharov VG, Hawkins J, Alferness C, Paone $\mathrm{G}$, et al. Passive epicardial containment prevents ventricular remodeling in heart failure. Ann Thorac Surg. 2000;70:1275-80.

25. Konertz WF, Shapland JE, Hotz H, Dushe S, Braun JP, Stantke K, et al. Passive containment and reverse remodeling by a novel textile cardiac support device. Circulation. 2001;104(Suppl 1):I270-5.

26. Kelley ST, Malekan RD, Gorman JH III, Jackson BM, Gorman RC, Suzuki Y, et al. Restraining infarct expansion preserves left ventricular geometry and function after acute anteroapical infarction. Circulation. 1999;1:135-42. 